\title{
Effect of vascular endothelial growth factor-C expression on lymph node metastasis in human cholangiocarcinoma
}

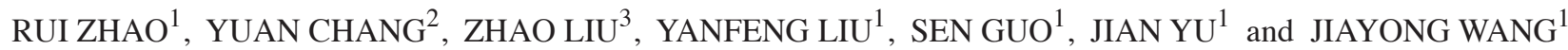 \\ ${ }^{1}$ Department of Hepatobiliary Surgery, Qilu Hospital, Shandong University, Jinan, Shandong 250012; \\ ${ }^{2}$ Department of Anesthesiology, Shandong Provincial Hospital Affiliated to Shandong University, Jinan, Shandong 250021; \\ ${ }^{3}$ Department of Hepatobiliary and Pancreatic Surgery, Jinan Central Hospital Affiliated to Shandong University, \\ Jinan, Shandong 250013, P.R. China
}

Received August 27, 2014; Accepted May 7, 2015

DOI: $10.3892 / 01.2015 .3309$

\begin{abstract}
The present study aimed to evaluate the expression of vascular endothelial growth factor C (VEGF-C) in human cholangiocarcinoma tissues and its role in metastasis in vitro. A total of 65 biopsy samples of cholangiocarcinoma, plus the FRH-0201 cell line, were investigated. The expression of VEGF-C in the human cholangiocarcinoma specimens was evaluated by immunohistochemistry (IHC). The effect of VEGF-C on tumor cell migration and proliferation was measured by MTT and Transwell assays in the FRH-0201 cell line. According to the IHC results, the biopsies of human cholangiocarcinoma were stained positively for VEGF-C, with a positive rate of $75.4 \%$ (49/65). Moreover, VEGF-C was expressed at a higher level in the patients with lymph node metastasis than in those without lymph node metastasis. In vitro, VEGF-C exhibited marked growth stimulation below the concentration of $5 \mathrm{ng} / \mathrm{ml}$ and was able to promote cholangiocarcinoma cell migration significantly. These findings suggested that VEGF-C may be a useful factor to predict lymph node metastasis in cholangiocarcinoma tissues and indicates that VEGF-C plays a significant role in proliferation and migration in cholangiocarcinoma cells.
\end{abstract}

\section{Introduction}

Cholangiocarcinoma is a highly malignant epithelial neoplasm arising from the bile duct epithelial cells or cholangiocytes of the intra- or extrahepatic biliary system. The incidence and mortality rates for cholangiocarcinoma are increasing worldwide. Due to the difficulty of forming an early diagnosis, more than two-thirds of cholangiocarcinomas have metastasis

Correspondence to: Dr Rui Zhao, Department of Hepatobiliary Surgery, Qilu Hospital, Shandong University, 107 Wenxi Road, Jinan, Shandong 250012, P.R. China

E-mail: ruizhao@yeab.net

Key words: cholangiocarcinoma, vascular endothelial growth factor- $\mathrm{C}$, metastasis, proliferation, immunohistochemistry and have no chance to benefit from surgical resection $(1,2)$. Lymphatic metastasis plays a significant role in the process of cholangiocarcinoma metastasis, and the hepatic portal lymph node is the most commonly affected node. Tumor metastasis is a complex and multi-stage process in which a number of cytokines are now known to be involved, particularly the vascular endothelial growth factor (VEGF) family (3-5).

VEGF-C has been implicated as being a potent stimulator of angiogenesis and lymphangiogenesis (6). By binding with VEGFR-2 and VEGFR-3, VEGF-C exerts its biological functions in various cell types. Recent studies revealed that VEGF-C can be expressed in several cancer cell types, including ovarian carcinoma (7), lung adenocarcinoma (8), breast cancer (9), and head and neck squamous carcinoma (10) cells, and that it can promote lymphatic metastasis progression, thus promoting tumor malignancy. Ishikawa et al (10) reported that the expression of VEGF-C is negative in early-stage gastric cancers due to the extremely low level of nodal metastasis, and Yonemura et al (11) showed that VEGF-C expression significantly correlates with lymph node metastasis in advanced-stage gastric cancers. However, few studies have been reported on the correlation between VEGF-C expression and an invasive phenotype in cholangiocarcinoma tissues and cells.

The present study evaluated whether VEGF-C can be a useful factor in predicting lymph node metastasis in human cholangiocarcinoma tissues and the role played by VEGF in mediating proliferation in the cholangiocarcinoma FRH-0201 cell line.

\section{Materials and methods}

Tissue collection and immunohistochemistry (IHC). A total of 65 patients with cholangiocarcinoma who were diagnosed and treated with curative surgery using a standard lymph node dissection, in Qilu Hospital of Shandong University (Jinan, Shandong, China) between 2012 and 2015, were included in the study. Written informed consent was obtained from all patients, and the study was approved by the ethics committee of Qilu Hospital of Shandong University. As normal controls, 5 cholangiolar biopsies from patients ( 3 female and 2 male; age range, 64-74 years) with a normal histology who underwent laparotomies were assessed. Fragments of cholangiocarcinoma 
and normal cholangiolar biopsies were processed for common histomorphology (hematoxylin-eosin and Masson's stains) and for IHC. For IHC, frozen cholangiocarcinoma tissue samples were stored at $-80^{\circ} \mathrm{C}$ until use. Cryostat sections were fixed in $4^{\circ} \mathrm{C}$ acetone (Beijing Zhongshan Biological Technology Ltd., Beijing, China) for $20 \mathrm{~min}$ and air-dried for $30 \mathrm{~min}$. Endogenous peroxidase activity was removed using $3 \% \mathrm{H}_{2} \mathrm{O}_{2}$ in methanol (Beijing Zhongshan Biological Technology Ltd.) for $10 \mathrm{~min}$. Subsequent to being washed three times with phosphate-buffered saline (PBS), the sections were incubated with an affinity-purified goat polyclonal $\mathrm{IgG}$ antibody against VEGF-C (catalog no. sc-7133; Santa Cruz Biotechnology Inc., Santa Cruz, CA, USA; dilution, 1:100) overnight at room temperature. Tissue sections were rinsed in PBS and then incubated with a linked biotinylated polyclonal rabbit anti-goat immunoglobulin secondary antibody (catalog no. E046601; Dako, Glostrup, Denmark) for $15 \mathrm{~min}$ at room temperature. Subsequent to rinsing with PBS, the sections were incubated with DAB and counterstained with hematoxylin (Beijing Zhongshan Biological Technology Ltd.). Negative controls were processed by omitting the primary antibody. The sections were observed using an Olympus BX53 fluorescent microscope (Olympus Corporation, Tokyo, Japan). Cases in which $>5 \%$ of the cells showed positive immunostaining for VEGF-C were judged to be positive.

Cell culture. The human hilar cholangiocarcinoma FRH-0201 cells were supplied by Dr Wu (Department of General Surgery, Qilu Hospital of Shandong University) and were maintained in Dulbecco's modified Eagle's medium (Hyclone, GE Healthcare, Logan, UT, USA) supplemented with $10 \%$ fetal bovine serum (FBS), $100 \mu \mathrm{g} / \mathrm{ml}$ streptomycina and $100 \mu \mathrm{g} / \mathrm{ml}$ penicillin (Zhejiang Tianhang Biological Technology Ltd., Hangzhou, China) and grown at $37^{\circ} \mathrm{C}$ in $5 \%$ carbon dioxide. The cells were starved for $4 \mathrm{~h}$ in medium without fetal bovine serum and then stimulated with VEGF-C at concentrations of 1, 5, 10, 50 and $100 \mathrm{ng} / \mathrm{m}$ for 24,48 and $72 \mathrm{~h}$, respectively, at $37^{\circ} \mathrm{C}$.

Proliferation assay. The effects of VEGF-C on the FRH-0201 cells were investigated by MTT assays (Promega Corporation, Madison, WI, USA). FRH-0201 cells at 85-100\% confluence were harvested, and then $5 \times 10^{3}$ cells per well were seeded onto a 96-well plate. The cells were incubated in $200 \mu \mathrm{l}$ medium in a humidified incubator. After $24 \mathrm{~h}$, the cells were stimulated with VEGF-C (Sino Biological Inc., Beijing, China; catalog no. 10542-H08H) at 1, 5, 10, 50 and $100 \mathrm{ng} / \mathrm{m}$ for 24,48 and $72 \mathrm{~h}$, respectively, and the medium was changed daily. Next, $20 \mu \mathrm{l}$ MTT solution was added and the cells were incubated for $4 \mathrm{~h}$, solubilized with $200 \mu \mathrm{l}$ DMSO (Amresco LLC, Solon, OH, USA) and agitated for $10 \mathrm{~min}$. The optical density (OD) values were examined at $570 \mathrm{~nm}$ using a Safire 2 microplate reader (Tecan Group Ltd., Männedorf, Switzerland). The cell survival percentages were calculated by dividing the mean OD of the VEGF-C-containing wells by that of the control wells.

Migration assay. Transwell chambers $(8-\mu \mathrm{m}, 24-w e l l$ insert; Corning Inc. Life Sciences, Tewksbury, MA, USA) were used for the migration assay. Briefly, $600 \mu 110 \%$ FBS-containing medium was added to the lower chamber and $1 \times 10^{5}$ cells
Table I. Summary of the clinicopathological features of 65 patients with cholangiocarcinoma.

\begin{tabular}{lr} 
Feature & Value \\
\hline Age, years (mean \pm standard deviation) & $55 \pm 8.5$ \\
Gender (male/female) & $38 / 27$ \\
Tumor location, $\mathrm{n}$ & \\
Hilar / Intrahepatic bile duct & 34 \\
Extrahepatic bile duct & 31 \\
Histological differentiation, $\mathrm{n}$ & \\
Well & 15 \\
Moderate & 19 \\
Poor & 31 \\
Lymphatic invasion, $\mathrm{n}$ & \\
Present & 42 \\
Absent & 23 \\
\hline
\end{tabular}
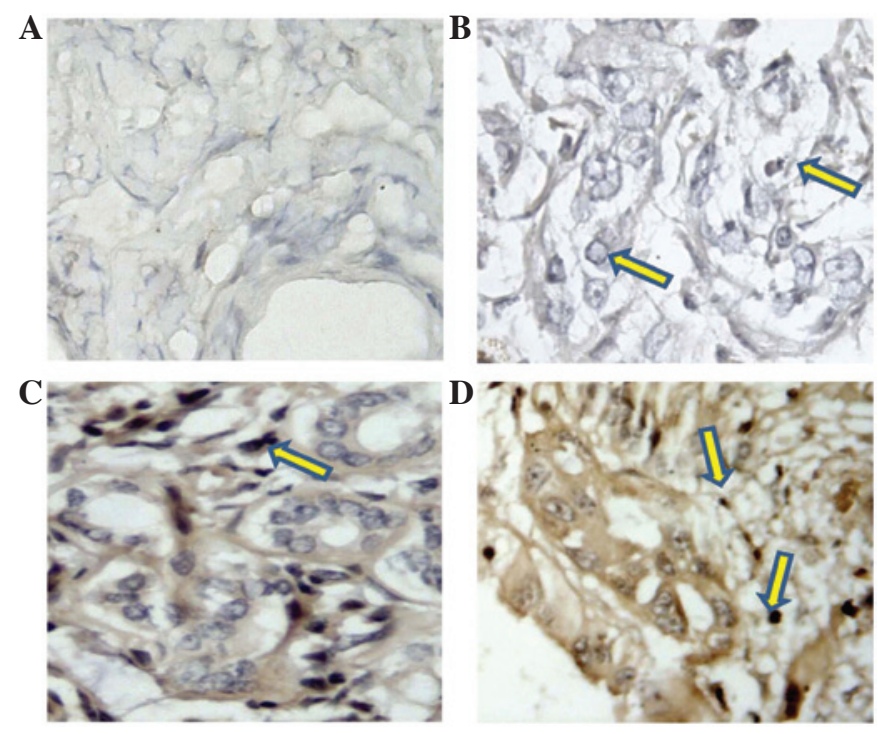

Figure 1. Immunohistochemical staining for vascular endothelial growth factor C (VEGF-C) in human cholangiocarcinoma. (A) Control specimen; (B) VEGF-C-negative cholangiocarcinoma specimen (arrows indicate VEGF-C was not observed); (C) cholangiocarcinoma specimen with weakly positive VEGF-C expression (arrow); and (D) cholangiocarcinoma specimen with strongly positive VEGF-C expression (arrows).

in $200 \mu \mathrm{l}$ serum-free medium were added to the upper chamber. The cells were incubated for $48 \mathrm{~h}$ at $37^{\circ} \mathrm{C}$, and the non-invading cells were then scraped off. Finally, the insert membranes were stained with $0.1 \%$ crystal violet (Amresco LLC) and the permeating cells were counted, with images captured under an Olympus CKX41 inverted microscope (Olympus Corporation). The migrated cells were counted in 10 random fields at $\times 200$ magnification. At least three randomly selected fields were counted, and the average number of cells was calculated per field.

Statistical analysis. Statistical analysis was performed using SPSS 15.0 software (SPSS, Inc., Chicago, IL, USA). Data were expressed as the mean \pm standard deviation, and 
Table II. Correlation between VEGF-C expression and pathological features.

VEGF-C expression, $\mathrm{n}$

\begin{tabular}{|c|c|c|c|c|}
\hline Feature & $\mathrm{n}$ & Positive & Negative & P-value \\
\hline Age, years & & & & $\mathrm{P}>0.05$ \\
\hline$\geq 60$ & 30 & 21 & 9 & \\
\hline$<60$ & 35 & 28 & 7 & \\
\hline Gender & & & & $\mathrm{P}>0.05$ \\
\hline Male & 38 & 28 & 10 & \\
\hline Female & 27 & 21 & 6 & \\
\hline Tumor location & & & & $\mathrm{P}>0.05$ \\
\hline Hilar/Intrahepatic & 34 & 26 & 8 & \\
\hline Extrahepatic & 31 & 23 & 8 & \\
\hline Tumor size, $\mathrm{cm}$ & & & & $\mathrm{P}>0.05$ \\
\hline$\geq 2$ & 21 & 18 & 3 & \\
\hline$<2$ & 44 & 31 & 13 & \\
\hline Differentiation & & & & $\mathrm{P}>0.05$ \\
\hline Well/moderate & 34 & 25 & 9 & \\
\hline Poor & 31 & 24 & 7 & \\
\hline Lymphatic invasion & & & & $\mathrm{P}<0.01$ \\
\hline Present & 42 & 36 & 6 & \\
\hline Absent & 23 & 13 & 10 & \\
\hline
\end{tabular}

VEGF-C, vascular endothelial growth factor C.

A

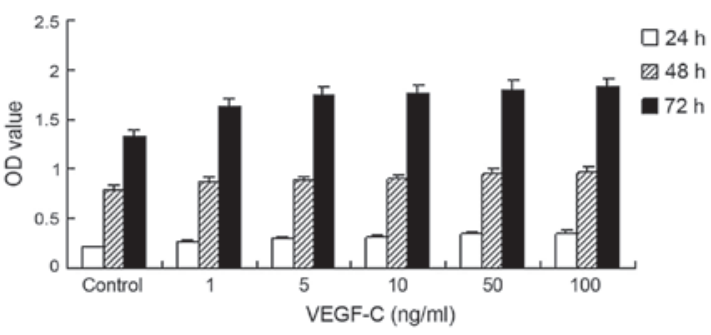

B

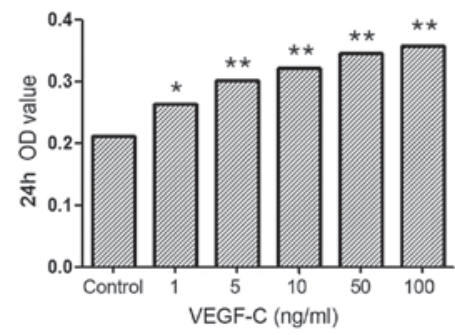

Figure 2. Effect of vascular endothelial growth factor C (VEGF-C) on the proliferation of FRH-0201 cholangiocarcinoma cells treated for different times, assessed by MTT assay. (A) The proliferation of cholangiocarcinoma cells treated with VEGF-C at concentrations of 1, 5, 10, 50 and $100 \mathrm{ng} / \mathrm{ml}$ for 24, 48 and $72 \mathrm{~h}$, respectively; (B) The proliferation of cholangiocarcinoma cells treated with VEGF-C at concentrations of 1, 5, 10, 50 and $100 \mathrm{ng} / \mathrm{ml}$ for $24 \mathrm{~h}$. Cell proliferation in the VEGF-C group was significantly different compared with that in the control group ( $\mathrm{P}<0.05,{ }^{* *} \mathrm{P}<0.01$ vs. control), but the rate of increase in cell proliferation slowed when VEGF-C concentrations exceeded $10 \mathrm{ng} / \mathrm{ml}$.

differences between groups were analyzed using Student's t-test. $\mathrm{P}<0.05$ was considered to indicate a statistically significant difference.

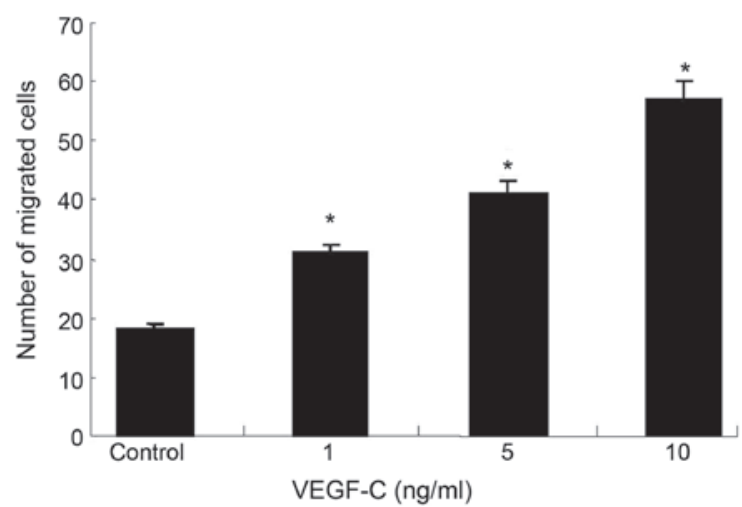

Figure 3. Effect of vascular endothelial growth factor C (VEGF-C) on the migaration of FRH-0201 cells, as determined by Transwell chamber assay. "P $<0.05$ vs. control.

\section{Results}

Expression of VEGF-C in cholangiocarcinoma tissues. The clinical and pathological characteristics of the 65 patients are summarized in Table I. The expression of VEGF-C was measured by IHC in the cholangiocarcinoma tissues and normal human bile duct specimens. Strong immunoreactivity was present for VEGF-C in the cholangiocarcinoma specimens, but minimal immunoreactivity was observed in the normal specimens (Fig. 1A and B). In the normal bile duct specimens, moderate- to low-level expression was only observed in $5 \%$ of the normal bile duct specimens. By contrast, 
high-level VEGF-C expression was observed in the majority of cholangiocarcinoma specimens, and the positive rate was 75.4\% (49/65) (Fig. 1C and D).

Association between the expression of VEGF-C and clinicopathological data. The association between clinicopathological data and the expression of VEGF-C is shown in Table II. No significant difference in tumor size was observed among the positive and negative expression types. There was also no significant difference between the expression of VEGF-C and age, gender and tumor location. However, VEGF-C was expressed at a significantly higher level in the patients with lymph node metastasis than in those without lymph node metastasis $(\mathrm{P}<0.01)$.

Effect of VEGF-C on the proliferation of FRH-0201 cells. The cell proliferation activities of the FRH-0201 cells were monitored by MTT assay. The results showed that VEGF-C exhibited marked growth stimulatory effects, compared with the control group, at concentrations of $1 \mathrm{ng} / \mathrm{ml}(\mathrm{P}<0.05)$ and $\geq 5 \mathrm{ng} / \mathrm{ml}(\mathrm{P}<0.01)$. However, when the concentrations of VEGF-C exceeded $10 \mathrm{ng} / \mathrm{ml}$, the rate of increase in cell proliferation slowed, and this increase was abolished by 100 ng/ml (Fig. 2).

VEGF-C promotes tumor cell migration. The effect of VEGF-C on FRH-0201 cell migration, a key determinant of malignant metastasis, was further examined. As shown in Fig. 3, ectopic high expression levels of VEGF-C led to significantly increased abilities of cell migration compared with the control cells $(\mathrm{P}<0.01)$, while more migrated cells were observed in the VEGF-C group compared with the control group $(\mathrm{P}<0.05)$.

\section{Discussion}

Tumor metastasis, an important factor in determining patient survival in cancer, occurs via the blood vascular and lymphatic systems to transport carcinogenic fluid and cells. A number of cytokines are now known to be involved in the process of tumor metastasis. VEGF-C is one of these factors, and is an essential predictor of tumor growth, lymphangiogenesis and metastatic behavior, as well as a variety of other significant pathologies $(12,13)$. However, there have been few studies on the expression of VEGF-C in cholangiocarcinoma, and the association between VEGF-C and lymph node metastasis has not been made entirely clear.

The present study clearly demonstrated the close association between VEGF-C expression and lymph node metastasis. Tumors with high expression levels of VEGF-C exhibited more remote lymph node involvement than those with low VEGF-C expression levels. The IHC study showed that VEGF-C was overexpressed in the cholangiocarcinoma tissues (positive ratio, 75.4\%), but the normal bile duct tissues were negative for this cytokine. Moreover, the concrete expression of VEGF-C was associated with lymphatic metastasis. In vitro, VEGF-C at 1 to $5 \mathrm{ng} / \mathrm{ml}$ exhibited marked growth stimulation compared with the control group. In Transwell chamber assays, VEGF-C led to significantly increased cell migratory ability. This is compatible with previous studies and suggests that VEGF-C induces tumor cell metastasis in cholangiocarcinoma $(14,15)$.

In the present study, certain clinically lymph node-negative cholangiocarcinoma specimens also showed VEGF-C expression. It is possible that the VEGF-C-positive cholangiocarcinoma patients without clinical lymph node metastasis in the present study may have exhibited lymph node metastasis at a microscopic level. Nevertheless, more importantly, the expression of VEGF-C was significantly higher in the lymph node-positive patients than in lymph node-negative patients, suggesting that the examination of VEGF-C expression in cholangiocarcinoma tissues may be an indicator of the presence of lymph node metastasis.

Taken together, these findings suggested that VEGF-C could promote proliferation and migration significantly in cholangiocarcinoma cells, and that there is a significant correlation between the expression of VEGF-C and lymphatic invasion. Hence, the targeting of VEGF-C may be a useful therapeutic strategy for certain patients with cholangiocarcinoma.

\section{Acknowledgements}

This study was supported by the National Science Foundation of China (grant no. 81302123) and the Promotive Research Fund for Excellent Young and Middle-aged Scientists of Shandong Province (grant no. BS2013YY029).

\section{References}

1. Patel T: Increasing incidence and mortality of primary intrahepatic cholangiocarcinoma in the United States. Hepatology 33: 1353-1357, 2001

2. Gores GJ: Cholangiocarcinoma: Current concepts and insights. Hepatology 37: 961-969, 2003.

3. Wu L, Li X, Ye L, et al: Vascular endothelial growth inhibitor is a negative regulator of aggressiveness and microvascular density in human clear cell renal cell carcinoma. Anticancer Res 34: 715-722, 2014.

4. Lozano-Santos C, Martinez-Velasquez J, Fernandez-Cuevas B, et al: Vascular endothelial growth factor A (VEGFA) gene polymorphisms have an impact on survival in a subgroup of indolent patients with chronic lymphocytic leukemia. PLoS One 27: e101063, 2014

5. Zhao R, Liu XQ, Wu XP, et al: Vascular endothelial growth factor (VEGF) enhances gastric carcinoma invasiveness via integrin alpha(v)beta6. Cancer Lett 287: 150-156, 2010.

6. Skobe M, Hamberg LM, Hawighorst T, et al: Concurrent induction of lymphangiogenesis, angiogenesis and macrophage recruitment by vascular endothelial growth factor- $\mathrm{C}$ in melanoma. Am J Pathol 159: 893-903, 2001.

7. Decio A, Taraboletti G, Patton V, et al: Vascular endothelial growth factor c promotes ovarian carcinoma progression through paracrine and autocrine mechanisms. Am J Pathol 184: 1050-1061, 2014

8. Liu J, Liu C, Qiu L, Li J, Zhang P and Sun Y: Overexpression of both platelet-derived growth factor-BB and vascular endothelial growth factor- $\mathrm{C}$ and its association with lymphangiogenesis in primary human non-small cell lung cancer. Diagn Pathol 9: $128,2014$.

9. Ciobanu M, Eremia IA, Crăiţoiu S, et al: Lymphatic microvessels density, VEGF-C and VEGFR-3 expression in 25 cases of breast invasive lobular carcinoma. Rom J Morphol Embryol 54: 925-934, 2013.

10. Ishikawa M, Kitayama J, Kazama S and Nagawa H: Expression of vascular endothelial growth factor C and D (VEGF-C and $-D)$ is an important risk factor for lymphatic metastasis in undifferentiated early gastric carcinoma. Jpn J Clin Oncol 33: 21-27, 2003.

11. Yonemura Y, Endo Y, Fujita H, et al: Role of vascular endothelial growth factor $\mathrm{C}$ expression in the development of lymph node metastasis in gastric cancer. Clin Cancer Res 5: 1823-1829, 1999. 
12. Kono M, Watanabe M, Abukawa H, Hasegawa O, Satomi T and Chikazu D: Cyclo-oxygenase-2 expression is associated with vascular endothelial growth factor $\mathrm{C}$ expression and lymph node metastasis in oral squamous cell carcinoma. J Oral Maxillofac Surg 71: 1694-1702, 2013.

13. Rubbia-Brandt L, Terris B, Giostra E, Dousset B, Morel P and Pepper MS: Lymphatic vessel density and vascular endothelial growth factor-C expression correlate with malignant behavior in human pancreatic endocrine tumors. Clin Cancer Res 10: 6919-6928, 2004.
14. Aishima S, Nishihara Y, Iguchi T, Taguchi K, Taketomi A, Maehara Y and Tsuneyoshi M: Lymphatic spread is related to VEGF-C expression and D2-40-positive myofibroblasts in intrahepatic cholangiocarcinoma. Mod Pathol 21: 256-264, 2008.

15. Wang WB, Li YH, Liu B, Wang HS, Cui AR and Zhnag XH: Correlation between PPARgamma and VEGF-C expression in extrahepatic cholangioadenocarcinoma (EHCAC) and their prognostic significance. Zhonghua Zhong Liu Za Zhi 31: 773-777, 2009 (In Chinese). 